\title{
ANÁLISIS DE MODELOS DEL TALENTO HUMANO APLICADOS EN ORGANIZACIONES PYMES
}

\author{
Analysis of human talent models applied at SMEs \\ Jorge Ariel Franco-López iD \\ MSc en Administración (MBA), Instituto Tecnológico Metropolitano. Medellín-Colombia, \\ jorgefranco@itm.edu.co \\ Janneth Bedoya-Zapata iD \\ Tecnóloga en Gestión Administrativa, Instituto Tecnológico Metropolitano. Medellín- Colombia, \\ jannethbz@gmail.com
}

\begin{abstract}
Cómo referenciar / How to cite
Franco-López, J. y Bedoya-Zapata, J. (2018). Análisis del talento humano aplicados en organizaciones pymes. Revista CEA, 4(7), 85-101. https://doi.org/10.22430/24223182.761
\end{abstract}

Recibido: 15 de agosto de 2017

Aceptado: 6 de octubre de 2017

\section{Resumen}

Las organizaciones tienen un aspecto clave que deben resolver los administradores cotidianamente $y$ es el talento humano; aspecto fundamental que hace permanecer, crecer y generar rentabilidad financiera y social. La investigación cualitativa desarrollada partió de una pregunta: ¿qué situaciones tienen las organizaciones pymes con respecto del talento humano? La propuesta teórica se fortaleció a partir de seis modelos de talento humano y una estrategia. Para el desarrollo metodológico se abordó un sistema categorial a partir de dos variables de estudio: exógenas con dos subcategorías; y endógenas con tres subcategorías. El instrumento para la recolección de información fue a través de diez entrevistas estructuradas hechas en organizaciones de Medellín. El análisis de los datos se realizó con la ayuda del software Atlas ti versión 7.5.10. Mediante un análisis hermenéutico, se concluye que las organizaciones analizadas carecen de gestión de personal, tarea soportada solo desde la administración. Estas organizaciones solo requieren empleados con capacidad operativa, los niveles de selección se establecen a través del referido, se evidencia incipientes procesos de capacitación y ausencia de planes carrera o de ascenso.

Palabras clave: gestión de personal, recursos humanos, talento humano.

\begin{abstract}
Organizations comprise a key aspect that administrators should deal with on a daily basis: human talent. It is fundamental and promotes loyalty, growth, and social and financial profitability. This qualitative research addressed the question "What situations do SMEs face regarding human talent?" For that purpose, six human talent models and a strategy enhanced the theoretical approach. The method adopted a categorical system based on two study variables: exogenous with two subcategories and endogenous with two other subcategories. The instrument to collect information comprised ten structured interviews conducted at organizations in
\end{abstract}


Medellín. The data were analyzed with the software Atlas ti version 7.5.10. By means of a hermeneutic analysis, it was concluded that the organizations under study lack human resource management, a task supported exclusively by the management. Such organizations only require employees with operational skills and recruitment is based on referrals. Besides, incipient training processes and no education or promotion plans were found.

Keywords: Personnel management, human resources, human talent.

\section{INTRODUCCIÓN}

La gestión del talento humano se considera al interior del discurso administrativo como parte fundamental de cualquier organización, en esa perspectiva es fundamental para toda gestión, conocer en profundidad los diferentes aspectos que influyen o determinan el comportamiento de tan importante recurso.

Las particularidades que representa cada persona hacen de cualquier estudio en talento humano una labor un tanto compleja; no existen recetarios o fórmulas que precisen sobre la mejor forma del cómo se debe comportar un empleado, trabajador o funcionario para obtener la mayor productividad posible en el desarrollo de las responsabilidades asignadas. Ahora, es claro que «Durante las últimas dos décadas se ha observado un gran interés por el desarrollo de la gestión del talento humano en las organizaciones, con el objetivo de estimular la innovación y creatividad» (Benjumea, Villa \& Valencia, 2016).

La investigación desarrollada comprende un análisis de la situación actual de los modelos de talento humano, entendiéndose según Chiavenato (2001), como un conjunto de políticas, conceptos y prácticas que aplican las distintas organizaciones en la gestión del personal, en nuestro caso, que aplican algunas pequeñas y medianas organizaciones (pymes) en la ciudad de Medellín. Se partió de una pregunta fundamental: ¿qué situaciones tienen las organizaciones pymes con respecto del talento humano?, a partir de allí se describe el problema.

Se usaron como referentes distintos modelos de gestión de personal que han marcado el campo de la administración: Harper y Lynch (Modelo de RH), Werther y Davis (Modelo de $\mathrm{GRH}$ ), Idalberto Chiavenato (Modelo de Administración de ARH), Beer et al. (Modelo de MGRH) Martha Alles (Modelo de Gestión por Competencias GPC), Sánchez, Martínez y Moreno (Modelo GCL), además, el plan Carrera, como estrategia para retener el talento humano, aportando valor a la gestión estratégica organizacional y consolidándose como una herramienta importante -si no decisiva- para alcanzar de manera exitosa las metas organizacionales, obtener los objetivos empresariales y la sostenibilidad en el largo plazo de las organizaciones que se aventuran en su implementación.

Para el desarrollo del objetivo se empleó una metodología en investigaciones de tipo cualitativa, fundamentada en un sistema categorial, el cual arrojó dos categorías de análisis: exógenas y endógenas. La primera categoría tuvo dos subcategorías, y la segunda categoría tuvo tres subcategorías, con lo cual se logró establecer el trabajo de campo. Como instrumento para tomar información se elaboró un cuestionario de preguntas para hacer diez entrevistas estructuradas aplicadas en igual número de organizaciones, reporte verbal brindado por los funcionarios que gestionan los departamentos de personal o propietarios de la organización que normalmente controla el personal. Con los hallazgos encontrados se utilizó el software Atlas ti7 versión 7.5.10, con el cual se 
obtuvieron veintidós mapas conceptuales, y, por último, para las consideraciones finales se utilizó el método hermenéutico de interpretación.

Se puede llegar a concluir que las pequeñas y medianas organizaciones de Medellín carecen de un organigrama claramente definido y consistente. La implementación de un modelo de gestión de talento humano es necesario y no basta con ser una tarea administrativa de soporte o transversal en la organización, porque direcciona procesos, los documenta y los coloca al servicio en la administración. Las competencias que debe tener un trabajador en el mercado de las pymes son básicamente operativas (del hacer), no requiere altos niveles de formación académica ni experiencia laboral. El proceso de selección y contratación es realizado, en casi todos los casos, directamente por la organización (pocas veces a través de terceros) y los requisitos, habilidades y aptitudes, es decir, las competencias que debe cumplir el aspirante son establecidos de acuerdo con las necesidades propias de la organización y su actividad comercial; esto no garantiza la permanencia o durabilidad del nuevo empleado.

El proceso de selección es vital para cualquier organización; debe verse desde una integralidad que posee la persona que ingrese, no es reclutar por el mero hecho de hacerse, es encontrar las personas con las competencias que satisfagan tanto los requisitos del cargo, como las necesidades de la organización. Las pymes son organizaciones con objetivos de corto plazo, donde solo viven el día a día, se carece de visión y misión organizacional que articulen los pocos recursos y canalice las mayores potencialidades, especialmente del recurso humano. Las pymes tienen un alto potencial, oportunidad y desarrollo, que es desaprovechado por la falta de visión, les permita no solo sobrevivir en el mercado, sino, además, posicionarse, avanzar y lograr éxito financiero y social.

\section{MARCO TEÓRICO}

Con relación a modelos en talento humano, menciona Rincón y Garzón (2012) las propuestas desarrolladas de autores, tal es el caso de Zerilli (1973), Sikula (1989), Koontz (1990), Werther y Davis (1992), Harper y Lynch (1992), Chiavenato (1995), Puchol (1995), Byars y Rue (1996), Cuesta-Santos (1999), Mesa-Espinosa (2000), Martínez (2002), Morales-Cartaya (2009). Se describen algunos modelos y estrategia en la Tabla 1.

Como referentes en distintas obras, se tiene en Ospina y Puentes (2011), un aporte académico sobre el análisis del talento humano en la organización Andina de Herramientas, la metodología de gestión por competencias observó que el aprendizaje organizacional permitía cumplir los objetivos tanto del talento humano como los de la organización. En Saldarriaga (2008), varios autores hacen la valoración sobre el talento humano, del cómo aumentar la productividad en la organización mediante modelos del capital humano, hace énfasis en los planes de formación de las potencialidades de las empleados. Por su parte Moscoso, en la industria Tucson (2007), menciona los planes de sucesión fundamentado por el análisis de competencias, para ello la organización debe hacer un óptimo reclutamiento, selección, contratación, e inducción, con esto garantizará que el talento humano sea considerado al interior del proceso de sucesión. Ríos (2011) desarrolla la propuesta en la Escuela de Ingeniería de Antioquia, cuando considera dos momentos claves en la gestión humana, reclutamiento y selección de personal. 
Tabla 1. Modelos de gestión del talento humano

Table 1. Human talent management models

\begin{tabular}{|c|c|c|}
\hline Autor & Planteamiento conceptual & Actividades de gestión \\
\hline $\begin{array}{l}\text { Modelo Recurso } \\
\text { Humano - RH. } \\
\text { Harper y Lynch } \\
\text { (Velázquez \& De } \\
\text { Miguel, 2001) }\end{array}$ & $\begin{array}{l}\text { Según Hernández, Fleites y Salazar (2011) } \\
\text { se desarrolla un plan estratégico a partir de } \\
\text { la previsión de necesidades, que presenta la } \\
\text { gestión de personal en la organización, se } \\
\text { busca la optimización en la gestión del } \\
\text { recurso humano, lo cual debe estar bajo } \\
\text { seguimiento para confrontar los resultados } \\
\text { obtenidos y las exigencias de la } \\
\text { organización frente a los objetivos, este } \\
\text { modelo es de carácter descriptivo, coloca } \\
\text { en evidencia todas las actividades en } \\
\text { relación con la gestión del talento humano, } \\
\text { con el fin de lograr un mejoramiento } \\
\text { significativo. }\end{array}$ & $\begin{array}{l}\text { Inventario de personal, selección, } \\
\text { evaluación del desempeño, planes de } \\
\text { comunicación, formación y de carrera, } \\
\text { estudios de clima y motivación, } \\
\text { organización del trabajo, ergonomía, } \\
\text { condiciones de trabajo y seguridad e } \\
\text { higiene, planificación estratégica de RH y } \\
\text { optimización de plantillas, sistemas de } \\
\text { pago, estimulación psicosocial, } \\
\text { auditoría, entre otras. "La satisfacción } \\
\text { de la demanda de recursos humanos } \\
\text { para la organización, se inicia con el } \\
\text { inventario del personal y la evaluación } \\
\text { del potencial humano y culminan con la } \\
\text { auditoría y el seguimiento a la } \\
\text { optimización de los recursos humanos» } \\
\text { (Hernández, Fleitas, \& Salazar, 2011). }\end{array}$ \\
\hline $\begin{array}{l}\text { Modelo Gestión } \\
\text { del Recurso } \\
\text { Humano - GRH } \\
\text { Werther y Davis } \\
\text { (Werther \& Davis, } \\
\text { 2008) }\end{array}$ & $\begin{array}{l}\text { Salinas (2012) plantea en el modelo cuatro } \\
\text { objetivos: Sociales: el departamento de } \\
\text { talento humano debe responder a una ética } \\
\text { y socialmente a los desafíos que presenta } \\
\text { dentro del contexto social, debe reducir al } \\
\text { máximo las tensiones o demandas } \\
\text { negativas que la sociedad ejerza sobre la } \\
\text { organización. } \\
\text { Organizacionales: toda organización busca } \\
\text { la productividad que garantice la } \\
\text { maximización del beneficio, por ello la GRH } \\
\text { tiene como labor contribuir a esta } \\
\text { situación. } \\
\text { Funcionales: la adaptabilidad es una } \\
\text { premisa fundamental del departamento de } \\
\text { talento humano, y con ello tener el mejor } \\
\text { personal para cumplir en forma eficiente el } \\
\text { objeto social de la organización. Personales: } \\
\text { la GRH debe contribuir a generar apoyo a } \\
\text { todos los empleados en sus metas, a tener } \\
\text { ambientes de trabajo adecuado } \\
\text { "En términos sencillos, la forma en que una } \\
\text { organización obtenga, mantenga y retenga } \\
\text { sus recursos humanos equivale a un factor } \\
\text { decisivo de su éxito o fracaso». (Werther \& } \\
\text { Davis, 2008) }\end{array}$ & $\begin{array}{l}\text { Hace énfasis en la alineación de las } \\
\text { actividades claves y trascendentes a } \\
\text { partir de dos planteamientos: 1) las } \\
\text { actividades y los objetivos como guías } \\
\text { del trabajo realizado en torno a la } \\
\text { gestión humana y su interacción y } \\
\text { retroalimentación constante; } 2 \text { ) los } \\
\text { retos y desafíos a los que se ven } \\
\text { sometidas las actividades programadas y } \\
\text { generadas en el área de gestión humana } \\
\text { hacia y desde el entorno organizacional. } \\
\text { (Ospina \& Puentes, 2011). }\end{array}$ \\
\hline $\begin{array}{l}\text { Modelo de } \\
\text { Administración de } \\
\text { Recurso Humano } \\
\text { - ARH }\end{array}$ & $\begin{array}{l}\text { La propuesta de Chiavenato, según } \\
\text { Martínez (2005) la estrategia que se } \\
\text { formule del talento humano en la } \\
\text { organización, debe generar un compromiso }\end{array}$ & $\begin{array}{l}\text { No hay una proyección estratégica del } \\
\text { talento humano, pero se destaca dentro } \\
\text { la relación de la organización un sistema, } \\
\text { el cual, con las entradas, procesos y }\end{array}$ \\
\hline
\end{tabular}




\begin{tabular}{l|l|l}
\hline Idalberto & de los trabajadores, de tal manera que se & salidas, arrojan resultados de la \\
Chiavenato & vuelvan socios estratégicos. Indica, & organización en su momento, lo cual \\
(Chiavenato, & además, que la ARH humano está & refleja cada día la realidad empresarial, \\
Administración de & constituida por subsistemas & y de esta forma se convierte en un \\
Recursos & interdependientes, adicionalmente cada & subsistema puente, entre otras áreas o \\
Humanos, 2001) & subsistema aplica unas políticas o & subsistemas de la organización que \\
& estrategias definidas para alcanzar los & permite realizar actividades claves para \\
& objetivos: alimentación del talento & alcanzar los objetivos propuestos.
\end{tabular}
humano, incluye la investigación de mercado de mano de obra, reclutamiento y selección. De aplicación del talento humano incluye el análisis y descripción de los cargos, integración o inducción, evaluación del mérito o del desempeño y movimientos del personal. De mantenimiento del talento humano incluye la remuneración, planes de beneficio social, higiene y seguridad en el trabajo, registros y controles del personal. Desarrollo del talento humano incluye los entrenamientos y los planes de desarrollo de personal, como el plan carrera. Control del talento humano abarca bases de datos, sistema de informaciones y auditoría. «Toda organización está compuesta de personas de las cuales dependen para alcanzar el éxito y mantener la continuidad. El estudio de las personas constituye la unidad básica de las organizaciones» (Chiavenato, 2001).

Modelo de $\quad$ El modelo MGRH indica la participación de Gestión del los empleados como aspecto contundente Recurso Humano para lograr las metas. Según Ospina y - MGRH Puentes (2011), los resultados se miden (Beer et al., 1989) con las cuatro «C» compromiso, competencia, congruencia y costos. A largo plazo genera dentro de la organización, bienestar social e individual, eficiencia empresarial, realimentación del sistema.

\begin{tabular}{|c|c|c|}
\hline & & \\
\hline $\begin{array}{l}\text { Modelo de } \\
\text { Gestión por } \\
\text { Competencias - } \\
\text { GPC } \\
\text { (Alles, 2011) }\end{array}$ & $\begin{array}{l}\text { "Las competencias son los conocimientos, } \\
\text { habilidades, actitudes, aptitudes, etc., que } \\
\text { hacen que el desarrollo de ciertas tareas y } \\
\text { actividades, así como el logro de } \\
\text { determinados resultados, sean } \\
\text { sobresalientes» (Lourdes, Casasús, Lara, } \\
\text { Liern, \& Pérez, 2008); en ese sentido, el } \\
\text { modelo GPC parte de la misión y la visión } \\
\text { organizacional, direccionando y articulando } \\
\text { todo el proceso para la gestión del talento } \\
\text { humanos por competencias, adicionando }\end{array}$ & $\begin{array}{l}\text { Las competencias son definidas, } \\
\text { establecidas y probadas por las } \\
\text { directivas, y luego gestionadas por } \\
\text { talento humanos. En esta última etapa, } \\
\text { las competencias requeridas para el } \\
\text { cargo son aplicadas al aspirante y a los } \\
\text { empleados de toda la organización. } \\
\text { Dentro de la creación de perfiles es } \\
\text { fundamental tener presente el } \\
\text { reclutamiento, selección } \\
\text { incorporación del personal, con base en }\end{array}$ \\
\hline
\end{tabular}




\begin{tabular}{|c|c|c|}
\hline & $\begin{array}{l}\text { que las características personales deben } \\
\text { tener una relación con el desempeño } \\
\text { sobresaliente, en un cargo/rol } \\
\text { determinado, conjugando con la estrategia } \\
\text { organizacional. } \\
\text { «El propósito de la implementación de un } \\
\text { modelo de competencias se relaciona con } \\
\text { dos ejes básicos vinculados entre sí: por un } \\
\text { lado, lograr que las personas que integren } \\
\text { la organización estén alineados con la } \\
\text { estrategia, y, por otro, desarrollar las } \\
\text { capacidades de las personas a fin que esta } \\
\text { alineación sea más efectiva y beneficiosa» } \\
\text { (Alles, 2008). }\end{array}$ & $\begin{array}{l}\text { esto se puede establecer los } \\
\text { conocimientos y competencias que } \\
\text { serán objeto de evaluación y análisis con } \\
\text { métodos o estrategias que brinden } \\
\text { también la posibilidad de identificar los } \\
\text { comportamientos, tales como, por } \\
\text { ejemplo, la entrevista por competencias. } \\
\text { El modelo se retroalimenta de manera } \\
\text { continua y cíclica, por esto siempre } \\
\text { estará en construcción. }\end{array}$ \\
\hline $\begin{array}{l}\text { Estrategia Plan } \\
\text { Carrera } \\
\text { (López, Gómez, \& } \\
\text { Betancourt, 2011) }\end{array}$ & $\begin{array}{l}\text { «El Plan de carrera debe ofrecer a un } \\
\text { individuo sobresaliente, de potencial } \\
\text { elevado, la posibilidad de encontrar un reto } \\
\text { profesional de manera permanente, que lo } \\
\text { conduzca a dar lo mejor de sí mismo» } \\
\text { (Aguilar, 2004). Sin embargo, un empleado, } \\
\text { para lograr ser sobresaliente, debe tener un } \\
\text { grado alto de motivación, entendido como } \\
\text { «un proceso complejo que canaliza } \\
\text { energías e influye en la forma como los } \\
\text { seres humanos persiguen sus fines o } \\
\text { intereses y los medios utilizados para } \\
\text { alcanzarlos» (López, Vélez \& Franco, 2017). } \\
\text { Ahora bien, al aplicar el concepto } \\
\text { motivación al interior de una organización } \\
\text { debe tenerse en cuenta que existen } \\
\text { factores intrínsecos y extrínsecos que } \\
\text { habilitan o deshabilitan la motivación del } \\
\text { empleado (Franco, Vélez, López \& Becerra, } \\
\text { 2017). }\end{array}$ & $\begin{array}{l}\text { «El concepto de planificación de carrera } \\
\text { dentro de una empresa es una práctica } \\
\text { que motiva al personal a permanecer en } \\
\text { ella, cuando esta práctica se realiza } \\
\text { sistemáticamente se muestra la política } \\
\text { de promociones como una realidad que } \\
\text { permitirá al individuo transitar un } \\
\text { camino desde el puesto actual hacia } \\
\text { otros de mayor categoría y de mayor } \\
\text { responsabilidad» (Zubillaga, 2010). } \\
\text { Es recomendable, desde la propuesta } \\
\text { del plan carrera, potencializar el talento } \\
\text { humano que se encuentra en la } \\
\text { organización. Los empleados esperan } \\
\text { tener ascensos como oportunidades } \\
\text { para surgir, y con ello tener un proyecto } \\
\text { de vida más claro, pero, además, } \\
\text { consecuentemente las organizaciones } \\
\text { llevan a sus empleados a una sana } \\
\text { competencia, con lo cual se logra } \\
\text { mayores niveles de productividad. }\end{array}$ \\
\hline $\begin{array}{l}\text { Modelo Gestión } \\
\text { por Competencias } \\
\text { Laborales } \\
\text { (GCL) } \\
\text { (Sánchez, } \\
\text { Martínez, \& } \\
\text { Moreno, 2017) }\end{array}$ & $\begin{array}{l}\text { La difícil situación económica cubana, a } \\
\text { partir de la década de los 90, hace } \\
\text { reflexionar a los autores, con lo cual } \\
\text { plantean un modelo para gestionar el } \\
\text { desarrollo integral del talento humano } \\
\text { fundamentado en competencias, buscando } \\
\text { mejorar los niveles de productividad en las } \\
\text { organizaciones. Hay cuatro factores } \\
\text { externos que vinculan las competencias de } \\
\text { talento humano, e interactúa con factores } \\
\text { propios de la organización. Es un modelo } \\
\text { sistémico y holístico. }\end{array}$ & $\begin{array}{l}\text { El enfoque del modelo integra factores } \\
\text { externos (regulaciones, desarrollo } \\
\text { científico técnico, clientes externos, } \\
\text { mercado de trabajo y capital humano), y } \\
\text { factores internos (estilos de dirección, } \\
\text { estrategia, cultura organizacional, } \\
\text { arquitectura empresarial). El capital } \\
\text { humano se integra a la arquitectura } \\
\text { organizacional en forma democrática y } \\
\text { participativa }\end{array}$ \\
\hline
\end{tabular}

Fuente: elaboración propia. 


\section{METODOLOGÍA}

Se aborda el trabajo desde la investigación cualitativa, donde el problema del conocimiento se visiona desde diferentes campos del saber: psicología organizacional, administración de personal, sociología de las organizaciones, entre otras. Se precisa, además, según Anguera (1986), que para llevar a cabo un tratamiento riguroso de la información en investigaciones cualitativas, dada las dificultades conceptuales, limitar la subjetividad, evitar sesgos en el análisis y tener rigor conceptual.

Para el constructo investigativo a nivel metodológico se utiliza el modelo planteado por Galeano (2011), donde la investigación cualitativa parte de categorías de análisis de conceptos sensibilizadores o también sistema categorial, que provean y orienten la aproximación al objeto de estudio; en los modelos teóricos y estrategias, denotan los autores que el entendimiento acertado del talento humano deberá existir una visión integral y holística, "comportamiento humano debe sustentarse bajo la premisa de un enfoque sistémico, proactivo, multidisciplinario, integral y participativo» (Aduna, García, y Chávez, 2017). En esa búsqueda de integralidad teórica que plantea Galeano (2011) con los modelos de Harper y Linch - RH (1992); Werther y Davis - GRH (1992); Chiavenato - ARH (1995); Beer - MGRH (1990); Alles - GPC (2005); estrategia Plan Carrera; Sánchez, Martínez y Moreno (2017), se destacan elementos que van desde manejo interno de la organización (endógenas o controlados), hasta el entorno social que interfiere en forma directa en el talento humano de la organización (exógenas o no controlados), como lo indica la Tabla 2.
En esa perspectiva, se direcciona el trabajo construyendo un sistema de dos categorías: y cinco subcategorías. La convalidación del cuestionario se hizo a partir de la búsqueda hecha en el marco teórico, el cual arrojó lo siguiente: categoría exógena, describe los aspectos sociales en el cual se encuentra inmerso el talento humano, con dos subcategorías, donde la organización no tiene un control (social), modelos $\mathrm{GRH}, \mathrm{RH}$, y la estrategia plan Carrera, son los pilares argumentativos. Por su parte, la segunda categoría, lo endógeno son los aspectos controlados por la organización, tiene tres subcategorías ubicadas desde los modelos ARH, GPS, GRH, MGRH, GCL y la estrategia plan Carrera. Ambas categorías se relacionan y perfilan el talento humano de la organización.

Para el tamaño de la muestra se utilizan los siguientes parámetros estadísticos: según Cámara de Comercio de Medellín (2018) hay 5736 organizaciones pequeñas y medianas (jurídicas y naturales) del sur de Medellín, lugar de la ciudad donde se tomó la muestra, nivel de confianza del $90 \%$, probabilidad de ocurrencia $50 \%$, de no ocurrencia $50 \%$, margen de error $30 \%$.

$$
\begin{aligned}
& n=\left(N \times Z^{2} \times p \times q\right) /\left(d^{2} \times(N-1)+Z^{2} \times p \times q\right) \\
& n=\left(5.736 \times 1,96^{2} \times 0,5 \times 0,5\right) \\
& \left(0,30^{2} \times(5.736-1)+1,96^{2} \times 0,5 \times 0,5\right) \\
& n=10 \text { entrevistas }
\end{aligned}
$$

El desarrollo de las entrevistas fue en las instalaciones de cada organización. La Tabla 3 muestra el tipo de organizaciones abordadas. La información fue bridada por jefes del área o departamentos de gestión de talento humano, administradores o el propietario. Se estableció un formato de 19 preguntas abiertas, las Tablas 4 y 5 describen las preguntas para cada subcategoría de las dos categorías. 
Tabla 2. Sistema categorial y subcategorías de análisis

Table 2. Categorical system and analysis subcategories

\begin{tabular}{|c|c|}
\hline Categoría & Subcategorías \\
\hline $\begin{array}{l}\text { Exógenas (social) } \\
\text { (Werther \& Davis - 2008) } \\
\text { (Harper y Linch - 1992) } \\
\text { Estrategia plan Carrera } \\
\text { (Sánchez, Martínez \& Moreno GCL 2017) }\end{array}$ & $\begin{array}{l}\text { 1. Entorno mercado laboral (Harper y Linch - 1992), (Werther } \\
\text { \& Davis - 2008), (Sánchez, Martínez \& Moreno, 2017) } \\
\text { 2. Organizaciones exitosas en gestión de personal en Medellín. } \\
\text { (Estrategia plan Carrera) }\end{array}$ \\
\hline $\begin{array}{l}\text { Endógenas (organizacional) } \\
\text { (Werther \& Davis - 2008) } \\
\text { (Chiavenato - 1995) } \\
\text { (Beer et al. - 1989) } \\
\text { (Alles 2005) } \\
\text { Estrategia plan Carrera, }\end{array}$ & $\begin{array}{l}\text { 3. Estructura salarial (Beer et al., 1989). } \\
\text { 4. Políticas o modelos en gestión de personal (todos los } \\
\text { modelos) } \\
\text { 5. Perfiles personales para reclutar, seleccionar, contratar, } \\
\text { socializar e inducir (Chiavenato, 1995), (Alles, 2005). }\end{array}$ \\
\hline
\end{tabular}

Tabla 3. Aspectos generales organizaciones entrevistadas

Table 3. General aspects of interviewed organizations

\begin{tabular}{l|l|l|c}
\hline Sector económico & Subsector & Objetivo social & $\begin{array}{c}\text { No. } \\
\text { trabajadores }\end{array}$ \\
\hline Industrial & Alimentos & Pulpa de fruta congelada & 10 \\
\hline Terciario & Administración & Arrendamiento propiedad horizontal & 23 \\
\hline Industrial & Alimentos & Transformadora de alimentos & 150 \\
\hline Industrial & Construcción & Soluciones de vivienda & 30 \\
\hline Industrial & Alimentos & Transformadora de alimentos & 15 \\
\hline Servicios & Bodegaje & Apoyo a la producción de cárnicos & 10 \\
\hline Industrial & Zapatos & Elaboración de calzado & 12 \\
\hline Industrial & Zapatos & Elaboración de calzado & 15 \\
\hline Servicios & Hotel & Prestación de servicios hoteleros & 23 \\
\hline Servicios & Financieros & $\begin{array}{l}\text { Prestadora de servicios financieros: ahorro y } \\
\text { crédito }\end{array}$ & 290 \\
\hline
\end{tabular}

Fuente: elaboración propia.

Tabla 4. Categoría exógenas formato de preguntas para las entrevistas estructuradas

Table 4. Exogenous category. Questions form for structured interviews

\begin{tabular}{|c|c|}
\hline Subcategoría & Preguntas \\
\hline $\begin{array}{l}\text { Entorno mercado } \\
\text { laboral }\end{array}$ & $\begin{array}{l}\text { 1. ¿Se podría pensar que el mercado de trabajo antioqueño tiene amplia } \\
\text { oferta para cualquier vacante que requiere la empresa? } \\
\text { 2. ¿La organización contrata directamente o utiliza un externo para esta } \\
\text { función (Cooperativa, fondo, pre cooperativa, empresa etc.)? } \\
\text { 3. ¿Considera que los trabadores y empleados de la organización son } \\
\text { productivos? } \\
\text { 4. ¿Hasta qué punto es importante el perfil del candidato en Facebook, } \\
\text { Linkedln o lo que escribe en Twitter? }\end{array}$ \\
\hline $\begin{array}{l}\text { Organizaciones exitosas } \\
\text { en gestión de personal } \\
\text { en Medellín }\end{array}$ & $\begin{array}{l}\text { 1. ¿Qué comentarios tiene sobre las medianas y pequeñas organizaciones de } \\
\text { Medellín con respecto a la gestión de personal? } \\
\text { 2. ¿Conoce la deserción de empleados que tiene la organización? ¿Qué piensa } \\
\text { sobre este resultado? }\end{array}$ \\
\hline
\end{tabular}

Fuente: elaboración propia. 
Tabla 5. Categoría endógenas formato de preguntas para las entrevistas estructuradas Table 5. Endogenous category. Questions form for structured interviews

\begin{tabular}{|c|c|}
\hline Subcategoría & Preguntas \\
\hline Estructura salarial & $\begin{array}{l}\text { 1. ¿'A través de qué parámetros establece la organización los salarios? } \\
\text { 2. ¿Existe un plan de incentivos salariales en la organización? } \\
\text { 3. ¿Cuáles son los tipos de contrato que tiene la organización? } \\
\text { 4. ¿Existe la figura de contrato por servicios, y de qué manera? } \\
\text { 5. ¿Cuál es el promedio de duración de los contratos en la organización? }\end{array}$ \\
\hline $\begin{array}{l}\text { Políticas o } \\
\text { modelos en } \\
\text { gestión de } \\
\text { personal }\end{array}$ & $\begin{array}{l}\text { 1. ¿Cuál es el procedimiento habitual de selección? } \\
\text { 2. ¿El departamento de talento humano de su organización se rige bajo un modelo } \\
\text { de gestión específico? Indique cuál. } \\
\text { 3. ¿Qué disciplina de recursos humanos cree que impulsa el desempeño y la } \\
\text { productividad en la organización? } \\
\text { 4. ¿'Tiene la posibilidad de obtener ascensos (cargos y salarios) los empleados } \\
\text { dentro de la organización? } \\
\text { 5. ¿Qué número de los empleados tienen un plan de desarrollo individual definido } \\
\text { 6. Cuando un empleado en la organización sabe que puede aspirar a tener mayores } \\
\text { responsabilidades con mejor sueldo, ¿está más motivado para desempeñar mejor } \\
\text { su trabajo? }\end{array}$ \\
\hline $\begin{array}{l}\text { Perfiles personales } \\
\text { para reclutar, } \\
\text { seleccionar, } \\
\text { contratar, } \\
\text { socializar e inducir }\end{array}$ & $\begin{array}{l}\text { 1. ¿Qué tipo de perfiles son los que más demanda o solicitan en su sector? } \\
\text { 2. ¿La organización cuenta con planes de carrera o desarrollo profesional, cuáles? } \\
\text { ¿Y cómo se ejecutan? } \\
\text { 3. ¿La organización invierte en la capacitación de los empleados? ¿En qué niveles o } \\
\text { cargos especialmente? } \\
\text { 4. ¿Cómo se compara el desempeño de los empleados que participan en programas } \\
\text { de formación opcionales en comparación con los que participan de manera } \\
\text { obligatoria? }\end{array}$ \\
\hline
\end{tabular}
Fuent: elaboración propia.

Para el análisis de la información se aplicó el software Atlas ti versión 7.5.10, programa para el análisis de información cualitativa que integra documentos textuales, datos de encuestas, datos bibliográficos, y se obtuvieron 22 mapas conceptuales; por último, se analiza cada categoría a través de un método hermenéutico para resolver las preguntas de investigación y los objetivos del trabajo.

\section{RESULTADOS}

La información obtenida en las 10 entrevistas estructuradas grabadas, se llevó a formato de Word, y a su vez al Atlas ti, desde allí se hace el constructo de las dos categorías y cinco subcategorías, ligándolas con las preguntas y las respectivas respuestas (Ver Figura 1). En total fueron veintidós mapas conceptuales obtenidos, de los cuales se muestran tres.

Se presenta una síntesis de cada categoría y subcategoría, como resultado de las entrevistas realizadas al personal encargado del talento humano en cada organización.

\section{Categoría exógena}

\section{Empresas exitosas en gestión de personal en Medellín}

Este tipo de organizaciones no tienen gestión de talento humano. Los niveles de deserción 
indicaron que el $60 \%$ no tienen deserción, el $20 \%$ sí la tienen y un $20 \%$ no sabe. Sin embargo, se puede indicar mediana estabilidad laboral en los trabajadores, porque el éxito de estas organizaciones es de corto y mediano plazo. (La Figura 2 indica hallazgos de la categoría exógena de la subcategoría entorno mercado laboral).

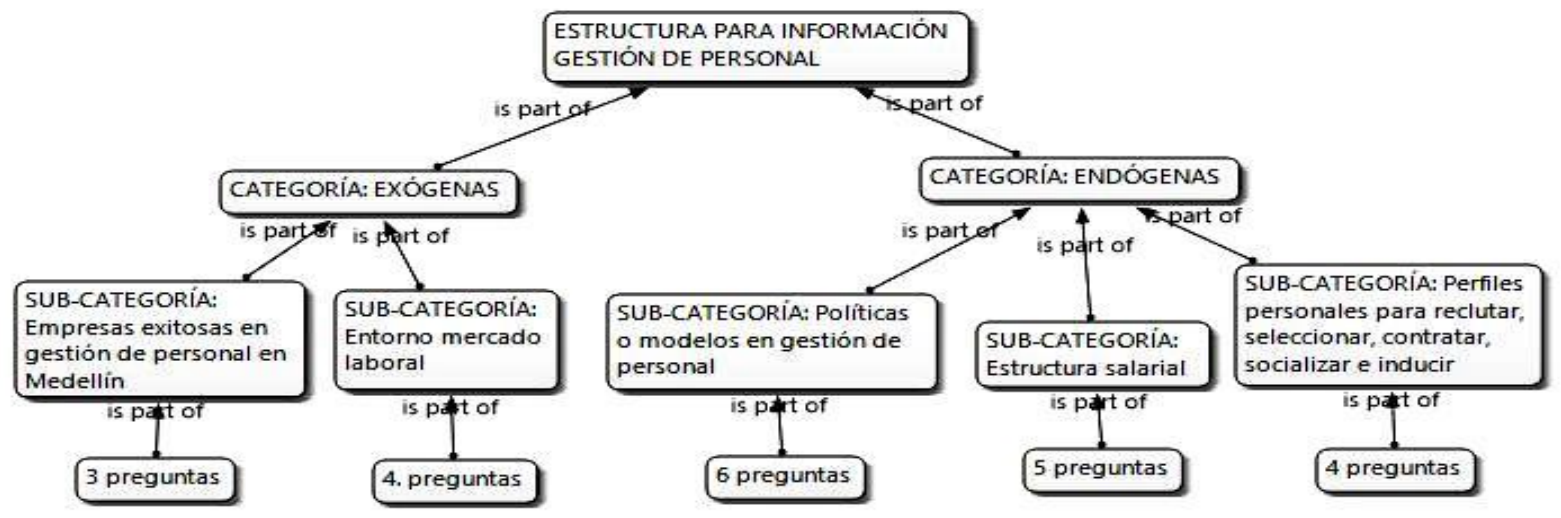

Figura 1. Estructura para las categorías y subcategorías

Figure 1. Categories and subcategories structure Fuente: elaboración propia a partir del análisis con el software Atlas ti versión 7.5.10.

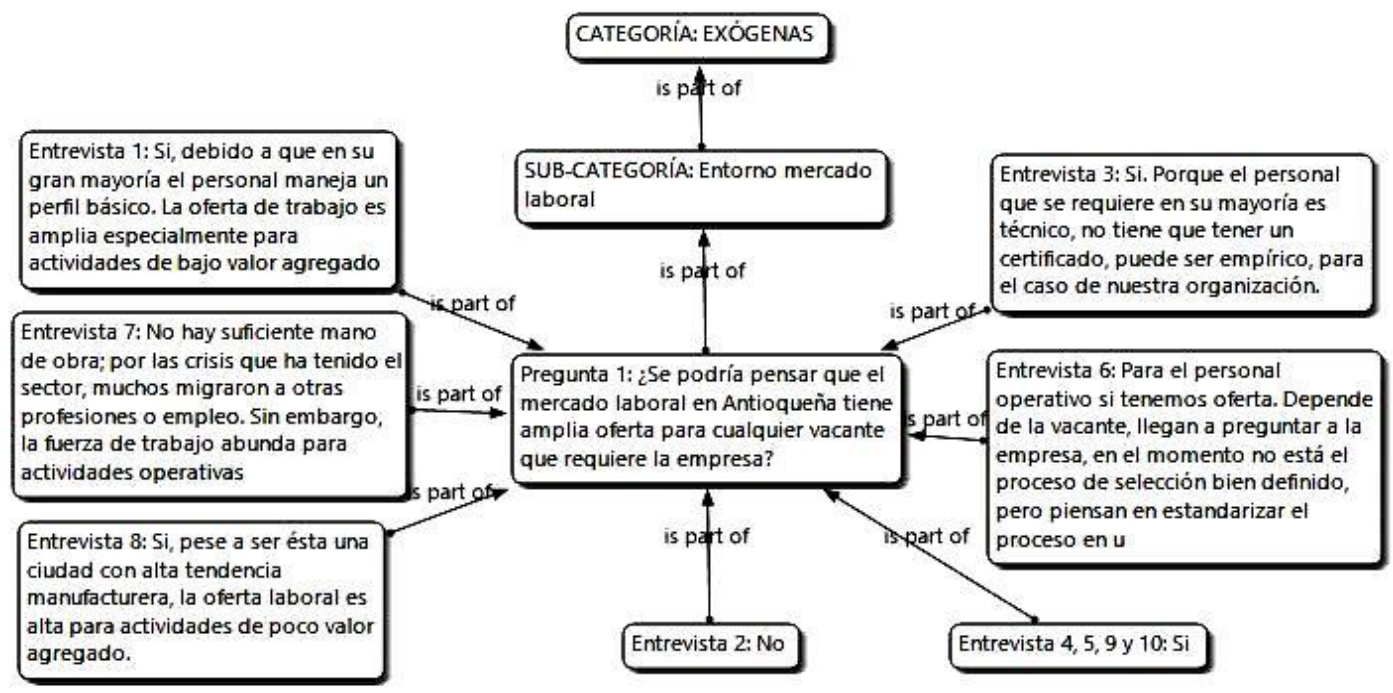

Figura 2. Categoría exógena, subcategoría entorno mercado laboral (pregunta 1)

Figure 2. Exogenous category. Subcategory labor market (Question 1)

Fuente: elaboración propia a partir del análisis con el software Atlas ti versión 7.5.10. 


\section{Categoría endógena}

\section{Entorno mercado laboral}

La oferta de trabajo en un país subdesarrollado, en la gran mayoría de los oficios, desborda la demanda, especialmente en las organizaciones pequeñas y medianas, donde el personal es de carácter operativo. Los niveles de contratación dependen de cada organización, sin embargo, la forma directa es la más utilizada, eliminando la cadena de intermediación. Referente a la productividad de los empleados, los jefes de personal y administradores respondieron que el $50 \%$ de los empleados son productivos, el 20 $\%$ que no, y un $30 \%$ en algunos casos. Estas organizaciones no utilizan redes sociales para buscar perfiles de candidatos a suplir vacantes laborales. (ver Figura 3).

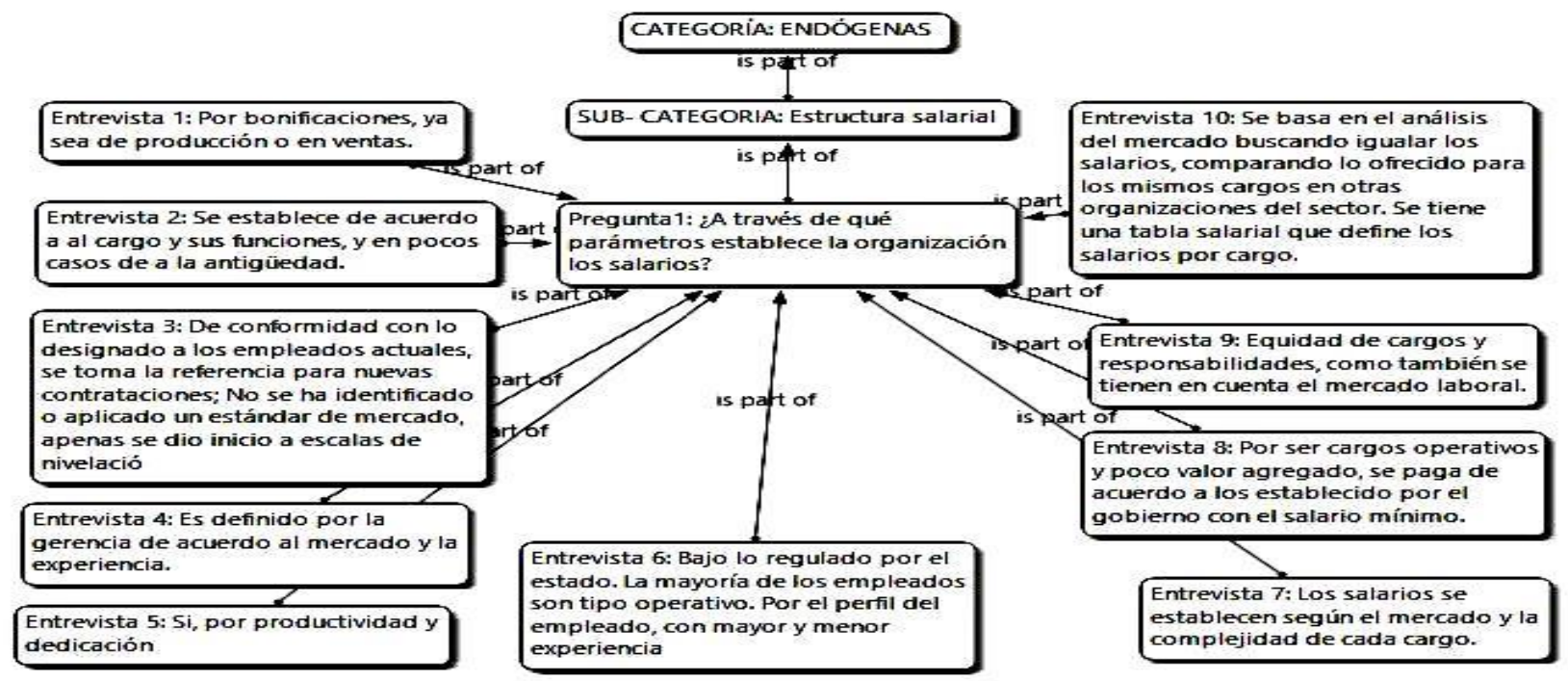

Figura 3. Categoría endógena, subcategoría estructural salarial (pregunta 1)

Figure 3. Endogenous category. Subcategory salary structure (Question 1)

Fuente: elaboración propia a partir del análisis con el software Atlas ti versión 7.5.10

\section{Políticas o modelos en gestión de personal}

Dentro de las políticas de reclutamiento se tiene a los recomendados como la modalidad más utilizada, además, allí se hace la selección de hojas de vida, pruebas, exámenes físicos, documentación, contratación, capacitación. Es de considerar que no todas las organizaciones hacen los mismos procedimientos. Ninguna organización tiene un modelo de gestión de talento humano que sea aplicado. Indicaron, referente a los ascensos que el $50 \%$ de las organizaciones entrevistadas dicen que sí lo tienen, el $30 \%$ que no, y un $20 \%$ en algunos casos.

\section{Estructura salarial}

Se cuenta con diferentes parámetros para establecer los salarios, tales como las condiciones del mercado, formas de contratación, funciones a desarrollar, entre otros. El $50 \%$ de las organizaciones tienen incentivos, el $30 \%$ no y el $20 \%$ no responde. Las modalidades de contratación son las permitidas por las leyes del derecho laboral: servicios, definido, indefinido. Los contratos de 
servicios no son la norma en este tipo de organizaciones. Los contratos término definido se han impuesto como modalidad.

\section{Perfiles personales para reclutar, seleccionar, contratar, socializar e inducir}

Las organizaciones entrevistadas se ubican en el segundo y tercer sector de la economía (industriales y de servicios), cada una de ellas recluta personal operativo, no requiere altos niveles de formación. Salvo un $10 \%$ de las entrevistadas, no existe plan Carrera, es decir, poca movilidad de ascenso del personal. Por su parte, la escasa capacitación brindada solo es de orden operativa.

En los resultados obtenidos se concluye que las organizaciones pequeñas y medianas carecen de modelos en gestión de talento humano. Se ajustan a las condiciones legales establecidas. El personal requerido es solo de orden operativo, no se tiene en su gran mayoría incentivos, capacitación y, obviamente, no hay planes de carrera. Las formas de selección se hacen en forma tradicional. Existe una gran oferta de trabajo por encima de la demanda, lo cual deprime los salarios reales. Todos estos factores en contra hacen de las organizaciones, entidades altamente vulnerables, incapaces de tener un verdadero capital humano generador de procesos innovadores.

\section{CONCLUSIONES}

Las organizaciones empresariales se han creado con el firme propósito de satisfacer necesidades para obtener rentabilidad financiera o social. Con el fin de lograr el objetivo, deben encaminar acciones concretas bajo un esquema lógico o racional de pensamiento y organización, donde se cuente con recursos y valores o principios que dictaminen el día a día. El modelo RH (1992), indicó que el talento humano se debe colocar al servicio de la organización para lograr productividad y mejores condiciones de vida al servicio de toda la organización, siguiendo una serie de actividades propias de un área que integre el talento humano como eje transversal de la organización, o un sistema puente (según el modelo ARH). Son muchas las inquietudes que se suscitan al interior de la organización, sobre el cómo se debe seleccionar, manejar, inventariar, comunicar, formar, evaluar, estimular, potencializar y auditar el personal de la organización. Las organizaciones investigadas presentaron asertividad para cumplir aspectos legales, pero carentes de integralidad que representa una real y genuina gestión del talento humano, aún se tiene una antigua visión organizacional, la cual es que, al involucrar recursos en esta área, representa un gasto y no un costo, lo cual es mencionado dentro del modelo MGRH, como una de las palabras claves que miden los resultados.

Ahora, para el objeto de estudio se observó que las pequeñas y medianas organizaciones de Medellín carecen de una estructura organizacional claramente definida y consistente. Contar con el mínimo de personal no genera la necesidad de crear una división jerárquica, sin embargo, la implementación de un modelo de gestión de talento humano es necesaria, y no basta con ser una tarea administrativa de soporte, porque direcciona procesos, los documenta y los coloca al servicio en su administración independiente de quién lo haga. El dinamismo actual de las organizaciones requiere adaptarse a las exigencias cambiantes de los mercados de competencia (modelo MGRH). "Los hombres pasan y las instituciones quedan», ello indica que la normalización es vital porque forma parte en la gestión del conocimiento. Según el modelo GRH, una gestión del talento humano es válida cuando cumple los objetivos sociales (categoría exógena), organizativos, funcionales y personales (categoría endógena). En ese sentido, el talento humano debe responder a 
aspectos éticos y sociales, los cuales son complejos en su buen funcionamiento cuando existe una estructura social desarticulada, con poca formación académica, ciudadana y en principios de vida.

Las competencias que debe tener un empleado en el mercado de las pymes son básicamente operativas (del hacer), no requiere altos niveles de formación académica y poca experiencia laboral, se presenta un mercado de trabajo donde la oferta de está siempre por encima de la demanda, en ese sentido se presentan bajos salarios reales, siendo relevante anotar que el grado de productividad del personal contratado no satisface en su totalidad las expectativas del empleador. ¿Qué genera lo anterior?, alta rotación de personal, ¿cómo se puede disminuir?, las organizaciones deben desarrollar estrategias pertinentes propias de la gestión de talento humano, esto asegura permanencia y permite acrecentar el capital humano, tratando de generar una integralidad para hacer de todos los empleados socios estratégicos (modelo ARH). Solo allí se presenta productividad, que sería el fin último de la organización, pero, además, que retribuya en equidad para los empleados con una remuneración salarial alta, incentivos, mejoramiento y lo ideal, un plan Carrera que lleve a ese empleado a ser cada vez más reconocido. "Si los empleados perciben que la organización invierte en ellos y los considere factores estratégicos, estos responderán con comportamientos positivos orientados a la consecución de los organizativos organizativos» Kuvaas and Dysvik (2010), citado por Benjumea et al. (2016).

El proceso de selección y contratación es realizado, en casi todos los casos, directamente por la organización (pocas veces a través de terceros) y los requisitos, habilidades y aptitudes que debe cumplir el aspirante, establecidas de acuerdo con las necesidades propias de la organización. Al respecto indicó el modelo GPC, que lo ideal es rodearse de empleados que estén alineados a la estrategia, para desarrollar las capacidades de acuerdo con las competencias que el empleado tenga, es decir, una óptima selección es aquella que integra estrategia, rol y competencia. Bajo esta perspectiva, se puede pensar el por qué se presenta deserción y despidos, en las medianas y pequeñas organizaciones, cuando esto representando altos costos para la organización, ¿será que la selección no es atravesada por un modelo de competencias? ¿Se contrata el personal por mera intuición o corazonadas? El proceso de selección es vital, debe verse desde una integralidad que debe poseer la persona que ingrese, no es reclutar por el mero hecho de hacerse, es encontrar las personas con las competencias que satisfagan las necesidades de la organización. Ahora, no es suficiente con lo anterior, la organización debe garantizar procesos posteriores, tal es el caso de la inducción, donde se adapta al trabajador al nuevo clima y ambiente laboral, se le enseña la cultura organizacional que seguirá teniendo mientras este en ese contexto. Otro proceso posterior que es fundamental es la capacitación, y es uno de los problemas de las pymes. Ahora, ¿por qué es importante la capacitación de los empleados? Es claro, en mercados de las pymes donde lo característico es competir donde existen sobreofertas, la empresa tendrá que encontrar la estrategia diferenciadora que le permitan mantenerse en el mercado, solo hay una posibilidad: talento humano capacitado, bien formado, y motivado.

De las formas de contratación se puede argumentar que representa un factor extrínseco motivacional para los trabajadores, ¿hasta qué punto?, es un gran motivador de estabilidad para el talento humano, genera cierto grado de estabilidad para el trabajador, cuando el contrato se establece en forma de término indefinido o definido, pero cuando el contrato es por servicios, la situación es 
desfavorable totalmente, porque los niveles de remuneración no satisfacen ni las mínimas condiciones de vida. Ahora, esta situación de los contratos también debe ser analizada desde la perspectiva del empleador, la visión remunerativa que se establece en el contrato debe ser proporcional al aporte en términos de productividad que genere el trabajador (salario vital), en esto se presenta serios cuestionamientos, porque las pymes son unidades productivas caracterizadas por ser ineficientes e ineficaces, en ese sentido, los rendimientos marginales del capital (beneficios) y del trabajo (salarios) son bajos. Esto es una gran disyuntiva de todas las pymes en unos lugares más que otros.

Las pymes son organizaciones con objetivos de corto plazo, donde viven el día a día, carecen de visión y misión que articulen los pocos recursos y canalice las mayores potencialidades, especialmente del talento humano. La gestión del talento humano es uno de los bastiones básicos sobre los cuales debe descansar el óptimo desarrollo de la organización, hoy los activos intangibles son el capital más importante que debe tener cualquier organización (capital humano), y en esa dirección deben encaminarse las pymes para obtener un crecimiento y rentabilidad.

Dentro de los muchos modelos se tienen el basado en competencias (GPC), se considera una propuesta interesante para ser exitosos en el manejo del personal, porque ubica el talento humano en los distintos componentes de la gestión: saber, saber hacer, saber estar, querer hacer y poder hacer. Determina al personal de acuerdo a cada competencia en la función adecuada.

Es fundamental capitalizar por encima de cualquier recurso, el talento humano. Una de las estrategias es el "plan Carrera», el cual tiene bondades por la movilidad de ascenso que tiene el personal, representando un incentivo importante para disminuir la deserción del personal, sin embargo, se ve complejo para el caso de las pymes, por tener estructuras productivas y organizacionales pequeñas, lo cual impide la movilidad del personal. Los ascensos que son incentivos al personal deberán ser llenados en las pymes por otras políticas de reconocimiento, tales como bonificaciones monetarias, tiempos de descanso, menciones honorificas, etc. Se debe destacar que aun siendo las pymes organizaciones con pocos recursos, las administraciones de ellas deben contener más planeación, el modelo $\mathrm{RH}$, indica que el caso de la gestión de personal requiere efectuar análisis en cada puesto de trabajo para definir las funciones, fundamentado por los niveles de competencia que tenga cada trabajador, mantener en alto la motivación a través de un buen clima laboral, evaluaciones de desempeño para cada gestión, planes de capacitación y estimulaciones psicosocial entre otras.

Las pymes tienen un alto potencial, oportunidad y desarrollo, que es desaprovechado por la falta de visión, estructura, organización y herramientas que permita no solo sobrevivir en el mercado si no posicionarse, avanzar y lograr mayor reconocimiento en el sector. Generalmente las pequeñas y medianas organizaciones se estancan, terminan fortaleciendo su sentido de supervivencia e inhabilitándose en su capacidad de explorar y desarrollar sus fortalezas.

Finalmente, la investigación desarrollada es un tema inagotable, siempre quedará en punta, porque el objeto de estudio presenta características particulares, los contextos culturales, sociales, políticos, económicos, varían, y establecer formas de comportamiento en el manejo del talento humano de acuerdo a fórmulas únicas será inapropiado. Con respecto del estudio 
desarrollado tuvo un limitante y fue encontrar información, muchas organizaciones ni siquiera tienen empleados que se dedican a la gestión del talento humano, más allá de los propietarios, en ese sentido, al ofrecer información tenía sesgos, la cual fue necesario depurar a través de la confrontación de otras fuentes, o en defecto, consideran que entregar información de este tipo es inapropiado y que puede ser mal utilizada.

Se referencian múltiples estudios de la temática en modelos y gestión del talento humano, Raeder (2018) sugiere que la temática sea abordada desde el capital humano $(\mathrm{CH})$, cualquier gestión del talento humano tiene que ver con los niveles de competencia que tenga el $\mathrm{CH}$ contrato en la organización. Jackson, Shuler and Jiang (2014) indicaron que el estudio del talento humano debe tener una mirada sistémica, para abordar múltiples intereses, que incluirían temas, tales como, gestión de la innovación, sostenibilidad ambiental, globalización, amplia competencia.

En Cassel, Nadin, Gray, y Clegg (2002), trabajo realizado en pymes, llegan a conclusiones parecidas a las nuestras, cuando existe una gran diversidad organizacional y se discute el modelo en la gestión del talento humano.

\section{REFERENCIAS}

Aguilar, I. (2004). El Recurso Humano frente a la Globalización. Civilizar, 13. Recuperado de http://repository.usergioarboleda.edu.co /handle/11232/190

Alles, M. (2008). Dirección estratégica de recursos humanos ( $2^{\mathrm{a}}$ ed.). Argentina: Ediciones Granica S.A. Recuperado de https://bit.ly/2joV8oo
Alles, M. (2011). Selección por competencias. Argentina: Granica S.A. Recuperado de https://books.google.com.co/books?id=J qzDDQAAQBAJ\&printsec=frontcover\&hl= es\#v=onepage $\& q \& f=$ false

Anguera, T. (1986). La investigación cualitativa. Educar, 10, 23-50. Recuperado de http://educar.uab.cat/article/download/ 461/442.

Benjumea, M., Villa, E., \& Valencia, J. (2016). Beneficio e impacto del teletrabajo en el talento humano. Resultados desde una revisión literaria. Revista CEA, 2(4), 59-73. Recuperado de http://revistas.itm.edu.co/index.php/revi sta-cea/issue/view/20

Cámara de Comercio de Medellín. (2018). Información empresarial. Medellín, Colombia. Recuperado de http://www.camaramedellin.com.co/site /Servicios-Empresariales/InformacionEmpresarial.aspx

Cassel, C., Nadin, S., Gray, M., \& Clegg, C. (2002). Exploring human resource management practices in small and medium sized enterprises. Personnel Review, 31(5-6), 671-692.

Chiavenato, I. (2001). Administración de recursos humanos (5 $5^{\mathrm{a}} \mathrm{ed}$.). (G. Villamizar, trad.). Colombia: McGrawHill. Recuperado de https://www.upg.mx/wpcontent/uploads/2015/10/LIBRO-27-

Administracion-de-RecursosHumanos.pdf

Galeano, E. (2011). Diseño de proyectos de la investigación cualitativa. Medellín, Colombia: Fondo Editorial Universidad Eafit. Recuperado de https://es.slideshare.net/juancarlosgome 
zjaramillo9/diseo-de-proyectos-en-la-invcualitativa-maria-eumelia-galeano

Hernández, I., Fleitas, S., \& Salazar, D. (2011). Particularidades de la gestión de los recursos humanos en empresas cubanas. Avanzada Científica, 14(1), 35-46. Recuperado de https://dialnet.unirioja.es/ejemplar/2795 75

Jackson, S., Shuler, R., \& Jiang, K. (2014). An Aspirational Framework for Strategic Human Resource Management. The Academy of Management Annals, 8(1), 156. Recuperado de https://bit.ly/2KwHTyc

Kuvaas, B. \& Dysvik, A. (2010). Exploring alternative relationships between perceived investment in employee development, perceived supervisor support and employee outcomes. Human Resource Management journal, 20(2), 138-156

López, H., Vélez, M., \& Franco, J. (2017). Percepciones acerca de la motivación docente en personal directivo de instituciones de educación secundaria en la zona metropolitana de Medellín, 2015. Electrónica Educare, 21(2), 1-23. Recuperado de https://dialnet.unirioja.es/servlet/articul o?codigo $=5919100$

López, M., Gómez, G., \& Betancourt, J. (2011). Factores que influyen en la participación de la mujer en cargos directivos y órganos de gobierno de la empresa familiar colombiana. Cuadernos de Administración, 24(42), 253-274. Recuperado de http://www.scielo.org.co/pdf/cadm/v24n 42/v24n42a12.pdf
Lourdes, C., Casasús, E., Lara, T., Liern, V., \& Pérez, J. (2008). Modelos flexibles de selección de personal basados en la valoración de competencias. Electrónica de Comunicaciones y Trabajos de Asepuma, 9, 101-122. Recuperado de https://dialnet.unirioja.es/servlet/articul o?codigo $=2888321$

Martínez, J. (2005). Las personas en la organización. Equidad y desarrollo, 3, 3543. Recuperado de https://revistas.lasalle.edu.co/index.php/ ed/article/view/372

Moscoso, T. (2007). Plan de sucesión para la empresa Tucson usando un modelo de competencias. Quito, Ecuador: Universidad Andina Simón Bolívar. Recuperado de http://repositorio.uasb.edu.ec/handle/10 644/813

Ospina, Z., \& Puentes, Y. (2011). Análisis y selección de un modelo de gestión Estratégico de Recursos humanos por competencias para la empresa Andina de herramientas. Santiago de Cali, Colombia: Universidad ICESI. Recuperado de http://bibliotecadigital.icesi.edu.co/biblio teca_digital/bitstream/10906/67387/1/a nalisis_seleccion_gestion.pdf

Raeder, S. (2018). Human resource management and career success: The supportive role of organizations. (I. f . psychologe, Ed.) Revista de Psicología Organizacional Aplicada, 49(1), 3-11. Recuperado de https://link.springer.com/article/10.1007 \%2Fs11612-018-0397-7

Rincón, C., \& Garzón, H. (2012). Sistema de Evaluación por Mérito. Bogotá, Colombia: Universidad EAN. Recuperado de http://repository.ean.edu.co/bitstream/h 
andle/10882/3585/Garz\%C3\%B3nHector 2012.pdf?sequence $=1$

Ríos, K. (2011). Propuesta de modelo para el reclutamiento y selección de empleados de la Escuela de Ingenieria de Antioquia. Medellín, Colombia: Institución Universitaria Ceipa. Recuperado de https://www.yumpu.com/es/document/v iew/14150110/propuesta-de-modelopara-el-reclutamiento-yseleccionnstitucion-

Saldarriaga, G. (2008). Gestión humana: tendencias y perspectivas. Estudios Gerenciales, 24(107), 137-159. Recuperado de http://www.scielo.org.co/scielo.php?scri pt=sci_arttext \&pid=S0123$59232008000200006 \& \operatorname{lng}=e n \& \operatorname{tng}=$ en

Salinas, C. (2012). Administración de personal: Modelo de Werther y Davis. Recuperado de

http://grupoadministraciondepersonas.bl ogspot.com.co/2012/10/modelo-dewerther-y-davies.html

Sánchez, A., Martínez, R., \& Moreno, J. (2017). Gestión por competencias laborales en el contexto de cambios políticos y económicos en Cuba. Innovar journal, 27(66), 169-184. Recuperado de http://www.scielo.org.co/pdf/inno/v27n 66/0121-5051-inno-27-66-00169.pdf

Velázquez, R., \& De Miguel, M. (2001). Modelos contemporáneos de gestión de recursos humanos. Ciencias Hoguín, 7(3). Recuperado de http://postgrado.una.edu.ve/desarrolloo rganizacional/paginas/velasquezlectura4 1.pdf

Werther, W., \& Davis, k. (2008). Administración de recursos humanos. El capital humano de las empresas ( $6^{a}$ ed.). (J. M. Gómez, Trad.) México: McGraw-Hill. Recuperado de

https://cucjonline.com/biblioteca/files/or iginal/c2f2989d851e80e2cc6aa0ebf3a54 cb0.pdf

Zubillaga, I. (2010). Planes de carrera, ¿mito o realidad? Observatorio Laboral Revista Venezolana, 3(5), 75-92. Recuperado de https://dialnet.unirioja.es/servlet/articul o?codigo $=3252753$ 
